\title{
Light-Induced Structural Changes in a Photosynthetic Reaction Center Caught by Laue Diffraction
}

Annemarie B. Wöhri et al.

Science 328, 630 (2010);

DOI: $10.1126 /$ science.1186159

This copy is for your personal, non-commercial use only.

If you wish to distribute this article to others, you can order high-quality copies for your colleagues, clients, or customers by clicking here.

Permission to republish or repurpose articles or portions of articles can be obtained by following the guidelines here.

The following resources related to this article are available online at www.sciencemag.org (this information is current as of June 4, 2012 ):

Updated information and services, including high-resolution figures, can be found in the online version of this article at:

http://www.sciencemag.org/content/328/5978/630.full.html

Supporting Online Material can be found at:

http://www.sciencemag.org/content/suppl/2010/04/27/328.5978.630.DC1.html

A list of selected additional articles on the Science Web sites related to this article can be found at:

http://www.sciencemag.org/content/328/5978/630.full.html\#related

This article cites $\mathbf{3 0}$ articles, 10 of which can be accessed free:

http://www.sciencemag.org/content/328/5978/630.full.html\#ref-list-1

This article has been cited by 4 articles hosted by HighWire Press; see:

http://www.sciencemag.org/content/328/5978/630.full.html\#related-urls

This article appears in the following subject collections:

Biochemistry

http://www.sciencemag.org/cgi/collection/biochem 


\section{Light-Induced Structural Changes in a Photosynthetic Reaction Center Caught by Laue Diffraction}

Annemarie B. Wöhri, ${ }^{1 *}$ Gergely Katona, ${ }^{2}$ Linda C. ${ }^{2}$ ohansson, ${ }^{2}$ Emelie Fritz, ${ }^{2}$ Erik Malmerberg, ${ }^{2}$ Magnus Andersson, ${ }^{1}$ ]onathan Vincent, ${ }^{3}$ Mattias Eklund, ${ }^{3}$ Marco Cammarata, ${ }^{4}$ Michael Wulff, ${ }^{4} \dagger$ Jan Davidsson, ${ }^{3}$ Gerrit Groenhof, ${ }^{5}$ Richard Neutze ${ }^{2} \ddagger$

Photosynthetic reaction centers convert the energy content of light into a transmembrane potential difference and so provide the major pathway for energy input into the biosphere. We applied time-resolved Laue diffraction to study light-induced conformational changes in the photosynthetic reaction center complex of Blastochloris viridis. The side chain of TyrL162, which lies adjacent to the special pair of bacteriochlorophyll molecules that are photooxidized in the primary light conversion event of photosynthesis, was observed to move 1.3 angstroms closer to the special pair after photoactivation. Free energy calculations suggest that this movement results from the deprotonation of this conserved tyrosine residue and provides a mechanism for stabilizing the primary charge separation reactions of photosynthesis.

A 11 photosynthetic reaction centers share a conserved functional core and are believed to have evolved from a single common ancestor. In the reaction center of the purple bacterium Blastochloris viridis $\left(\mathrm{RC}_{\mathrm{vir}}\right)(1)$, transmembrane subunits $\mathrm{H}, \mathrm{L}$, and $\mathrm{M}$ support four bacteriochlorophylls, two bacteriopheophytins, a bound menaquinone $\left(\mathrm{Q}_{\mathrm{A}}\right)$, a mobile ubiquinone $\left(\mathrm{Q}_{\mathrm{B}}\right)$, and a single nonheme iron, creating an electron transfer chain that spans the membrane (Fig. 1A). Photoexcitation of a strongly interacting pair of bacteriochlorophyll molecules, the "special pair" (P), promotes the movement of an electron to the primary and secondary quinone acceptors, $\mathrm{Q}_{\mathrm{A}}$ and $\mathrm{Q}_{\mathrm{B}}$, respectively (black arrows, Fig. 1A). After the reduction of $\mathrm{P}^{+}$from the tetraheme cytochrome $\mathrm{c}$ subunit (red arrows, Fig. 1A), the absorption of a second photon by the special pair triggers a second electron transfer to $\mathrm{Q}_{\mathrm{B}}{ }^{-}$, which is protonated from the cytoplasm and released as ubiquinol $\left(\mathrm{QH}_{2}\right)$ into the membrane. Coupled cyclic electron transfer reactions involving the integral membrane cytochrome $\mathrm{bc}_{1}$ complex and soluble cytochrome $\mathrm{c}_{2}$ ensure that

${ }^{1}$ Department of Chemical and Biological Engineering, Chalmers University of Technology, Box 462, SE-40530 Göteborg, Sweden. ${ }^{2}$ Department of Chemistry, Biochemistry and Biophysics, University of Gothenburg, Box 462, SE-40530 Göteborg, Sweden. ${ }^{3}$ Department of Photochemistry and Molecular Science, Uppsala University, Box 523, SE-75120 Uppsala, Sweden. ${ }^{4}$ European Synchrotron Radiation Facility, BP 220, Grenoble Cedex 38043, France. ${ }^{5}$ Computational Biomolecular Chemistry Group, Department of Theoretical and Computational Biophysics, Max Planck Institute for Biophysical Chemistry, Am Fassberg 11, D-37077 Göttingen, Germany.

*Present address: AstraZeneca Research and Development, Cell, Protein, and Structural Sciences, SE 43183 Mölndal, Sweden.

tPresent address: SLAC National Accelerator Laboratory, 2575 Sand Hill Road, Menlo Park, CA 94025, USA.

†To whom correspondence should be addressed. E-mail: Richard.Neutze@chem.gu.se

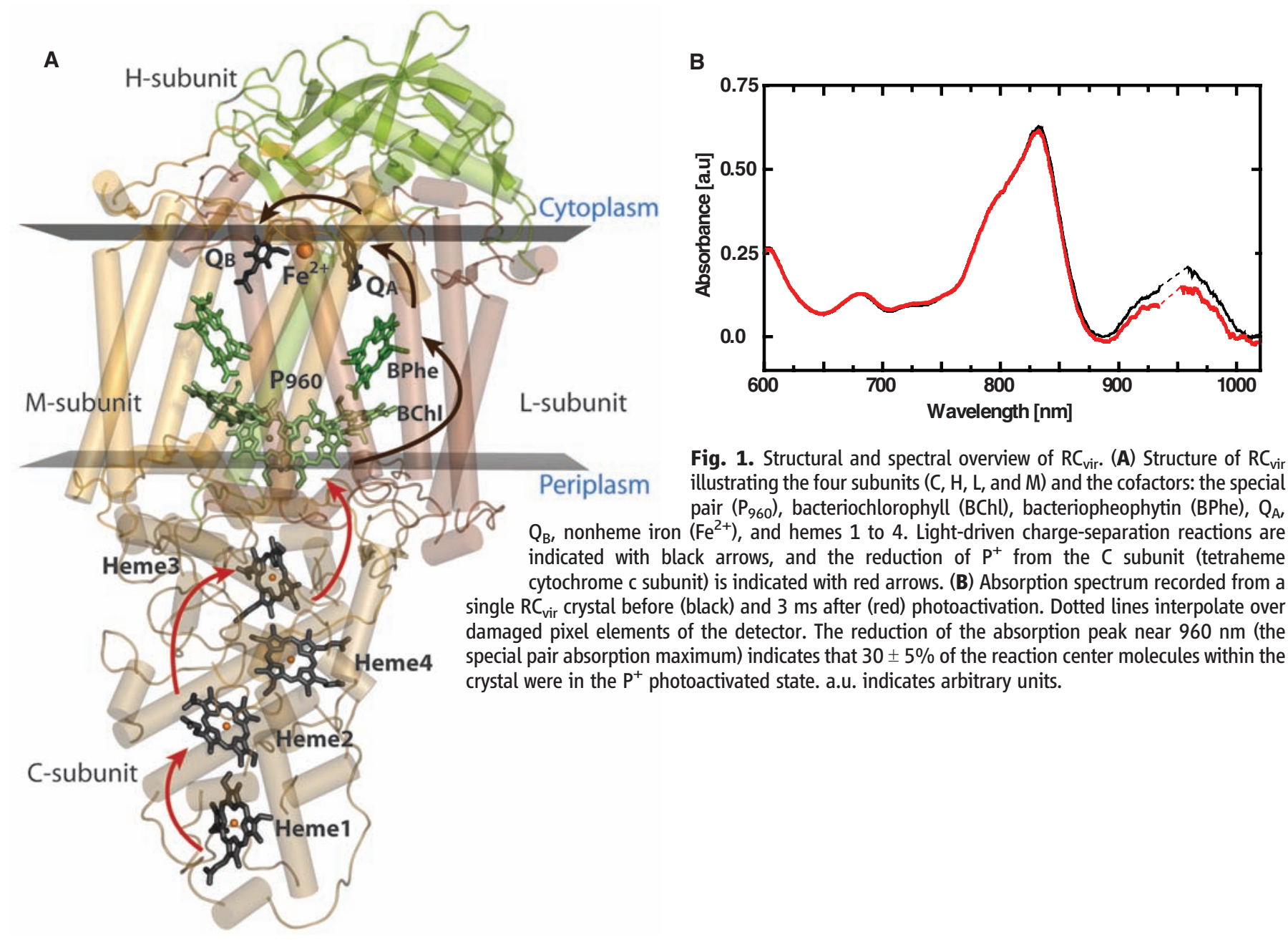


the tetraheme cytochrome c subunit is reduced from the periplasm (fig. S1). As a consequence, two protons are pumped for every photon absorbed, and the resulting transmembrane potential difference is primarily harvested by adenosine triphosphate (ATP) synthase to regenerate ATP.

Protein conformational changes are believed to participate in the function and regulation of photosynthetic reaction centers. X-ray diffraction studies of crystals of the Rhodobacter sphaeroides reaction center $\left(\mathrm{RC}_{\mathrm{sph}}\right)$ illuminated at room temperature before rapid freezing implicated a substantial movement of $\mathrm{Q}_{\mathrm{B}}$ upon its reduction to semiquinone (2). A concerted movement of the $\mathrm{H}$ subunit was also observed in crystals of the same reaction center after prolonged illumination with bright light (3). Time-resolved Laue diffraction provides a direct approach for observing protein conformational changes in real time at room temperature. This method has captured lightinduced electron density changes in myoglobin: carbon monoxide complexes $(4,5)$ and photoactive yellow protein $(6,7)$, although similar studies of a photosynthetic reaction center did not reveal any structural changes (8).

In this work, we used time-resolved Laue diffraction $(9)$ to probe light-driven conformational changes occurring at room temperature in
A
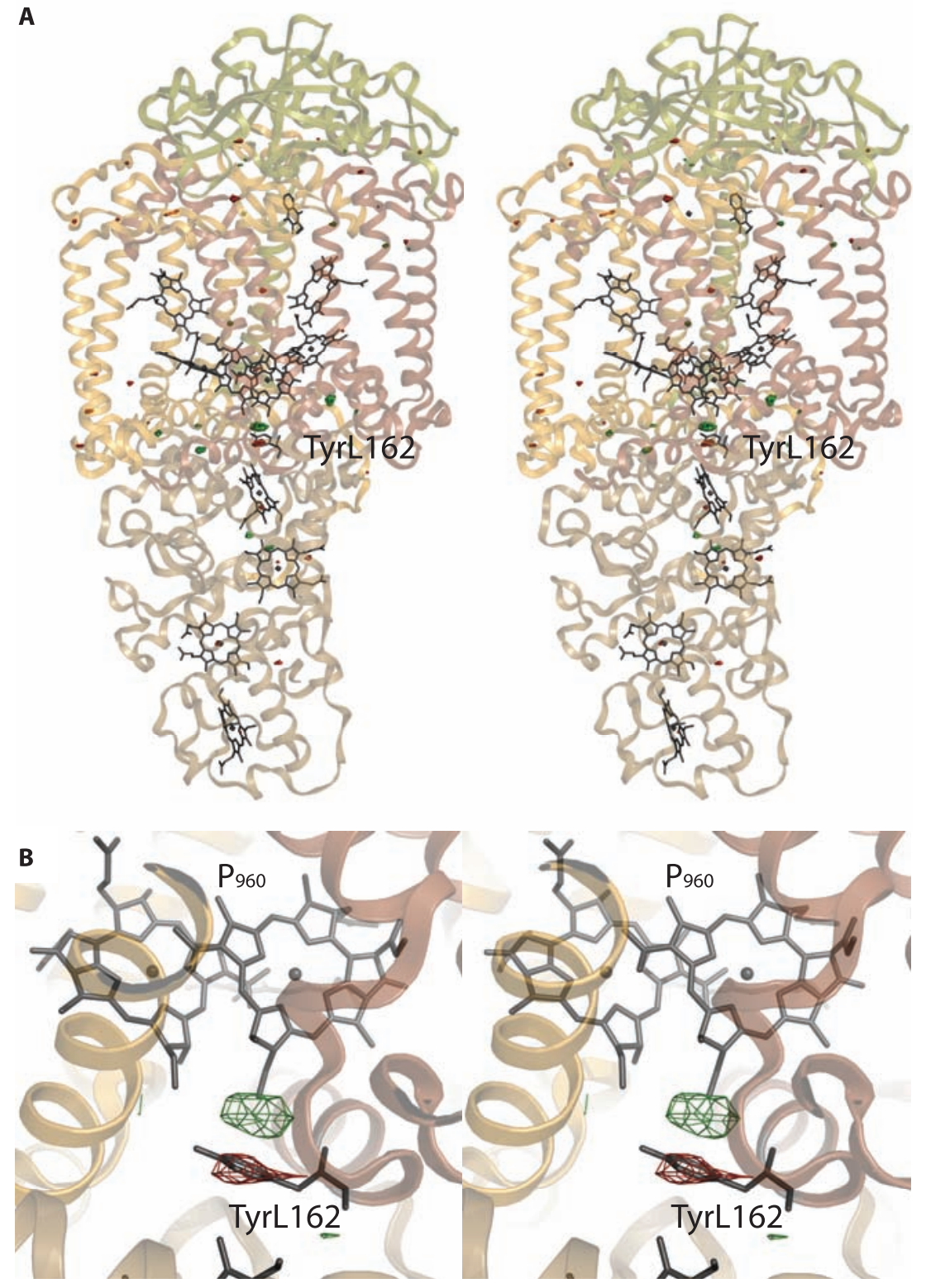

Fig. 2. Experimentally observed electron density changes in $\mathrm{RC}_{\mathrm{vir}}$ (A) Long-distance stereo overview of the $F_{\text {light }}-F_{\text {dark }}$ difference Fourier electron density map recorded $3 \mathrm{~ms}$ after laser photoactivation (green mesh, positive density; red mesh, negative density; contoured at $4.0 \sigma$ ). (B) Close-up stereoview of the difference Fourier map near the special pair. Complementary positive and negative electron density changes (contoured at $4.0 \sigma$ ) indicate the movement of TyrL162 toward $\mathrm{P}^{+}$.
$\mathrm{RC}_{\mathrm{vir}}$ crystals grown from a lipidic-sponge phase matrix (10). Time-resolved single-crystal microspectrophotometry (9) established that the photooxidized $\mathrm{P}^{+}$state persisted with $30 \pm 5 \%$ occupancy $3 \mathrm{~ms}$ after exposure to green light (Fig. 1B), and these crystals diffracted to $2.5 \AA$ resolution when exposed to a single polychromatic x-ray pulse train of $86-\mu$ s duration (fig. S2). Table S1 summarizes the Laue diffraction data and refinement statistics processed to $2.95 \AA$ resolution for both the resting state (laser off, $F_{\text {dark }}$ ) and $3 \mathrm{~ms}$ after photoactivation (laser on, $\left.F_{\text {light }}\right)$, and fig. S3 illustrates the $2 F_{\text {obs }}-F_{\text {calc }}$ electron density map. An overview of the experimental $F_{\text {light }}-F_{\text {dark }}$ difference Fourier electron density map is shown in Fig. 2A. As with an earlier Laue diffraction study on $\mathrm{RC}_{\text {vir }}(8)$, no significant difference density features arise to suggest a movement of ubiquinone within the $\mathrm{Q}_{\mathrm{B}}$ pocket, perhaps because of variable $\mathrm{Q}_{B}$ occupancy in this crystal form (10).

Both the strongest positive (green) and negative (red) difference electron density peaks (table S2) are associated with the side chain of $\mathrm{Tyr}^{162}$ of the L subunit (TyrL162) (Fig. 2B). Paired negative and positive difference density features are the fingerprint of conformational change and reveal that the side chain of TyrL162 reorients toward $\mathrm{P}^{+}$in the photoactivated state. These features are also the most reproducible difference density peaks observed in maps recovered from three independent crystals (table S2 and fig. S4A), and they persisted after diffraction data from all three crystals were merged (table S2 and fig. S4B). Theoretical difference Fourier maps calculated to $2.95 \AA$ resolution from the photoactivated and resting-state models reproduced these difference density features (fig. S5), and the crystallographic occupancy was $35 \pm 5 \%$ for the new conformation, consistent with spectroscopic characterization of the $\mathrm{P}^{+}$state (Fig. 1B).

TyrL162 is strictly conserved in purple bacterial reaction centers $(11,12)$ and lies between the special pair of bacteriochlorophylls and the highest potential heme $c_{559}$ (heme 3, Fig. 1A) of the cytochrome c subunit. Its side chain hydroxyl group forms a H-bond interaction with a water molecule (Wat501), which in turn is coordinated by two other water molecules and the hydroxyl group of SerM188 (Fig. 3A). Structural refinement of the photoactivated state (table S1) established that the hydroxyl oxygen of TyrL162 undergoes a $1.3 \AA$ movement toward $\mathrm{P}^{+}$(Fig. 3B), effectively bringing it into steric contact with the L-branch bacteriochlorophyll of $\mathrm{P}$ (fig. S6). This conformational change also creates new H-bond interactions of TyrL162 with the hydroxyl group of ThrM185 and Wat10 (Fig. 3B). A possible movement of Wat501 that would preserve its $\mathrm{H}$ bond to TyrL162 could not be refined because of spatial overlap with the restingstate model.

Photooxidation of the special pair changes the electrostatic environment of TyrL162, and this must drive its movement toward the transient 
positive charge, which implies that TyrL162 is negatively charged in the photoactivated state. Conversely, it is energetically unfavorable to bury an isolated charge within the low dielectric environment of a cellular membrane, such that the photooxidized special pair could be stabilized by the creation of a nearby phenolate anion. The transient formation of $\mathrm{H}$-bond interactions by TyrL162 with ThrM185 and Wat10 (Fig. 3B) would also stabilize its deprotonated form. Molecular dynamics trajectories demonstrate that the deprotonated form of TyrL162 adopts a confor- mation $1.1 \AA$ closer (on average) to the photooxidized special pair than when it is protonated (Fig. 3, C and D) and $1.3 \AA$ A closer than that adopted without photoactivation, consistent with our structural findings. We thus conclude that the photooxidation of $\mathrm{P}$ to $\mathrm{P}^{+}$changes the chemical environment of TyrL162 such that its deprotonated form becomes favored. Fourier transform infrared spectroscopy studies of electrochemically oxidized reaction centers have observed a difference signal at $1532 \mathrm{~cm}^{-1}$ for $\mathrm{RC}_{\mathrm{vir}}\left(1526 \mathrm{~cm}^{-1}\right.$ for $\left.\mathrm{RC}_{\mathrm{sph}}\right)$ that was interpreted as arising from conformational or
Fig. 3. Modeled lightinduced structural changes in $\mathrm{RC}_{\mathrm{vir}}$ and their dependence on the protonation state of TyrL162. (A) Resting-state conformation of TyrL162. A chain of water molecules (red spheres) stretches from TyrL162 toward the protein's surface via SerM188 and GluC254. (B) Refined movement of TyrL162 (blue) overlaid on its resting-state conformation (gray). In the photoactivated conformation, TyrL162 forms $\mathrm{H}$-bond interactions with ThrM185 and Wat10. (C) Snapshots from molecular dynamics trajectories showing a reorientation of TyrL162 toward $\mathrm{P}^{+}$ observed when TyrL162 is deprotonated (blue) but
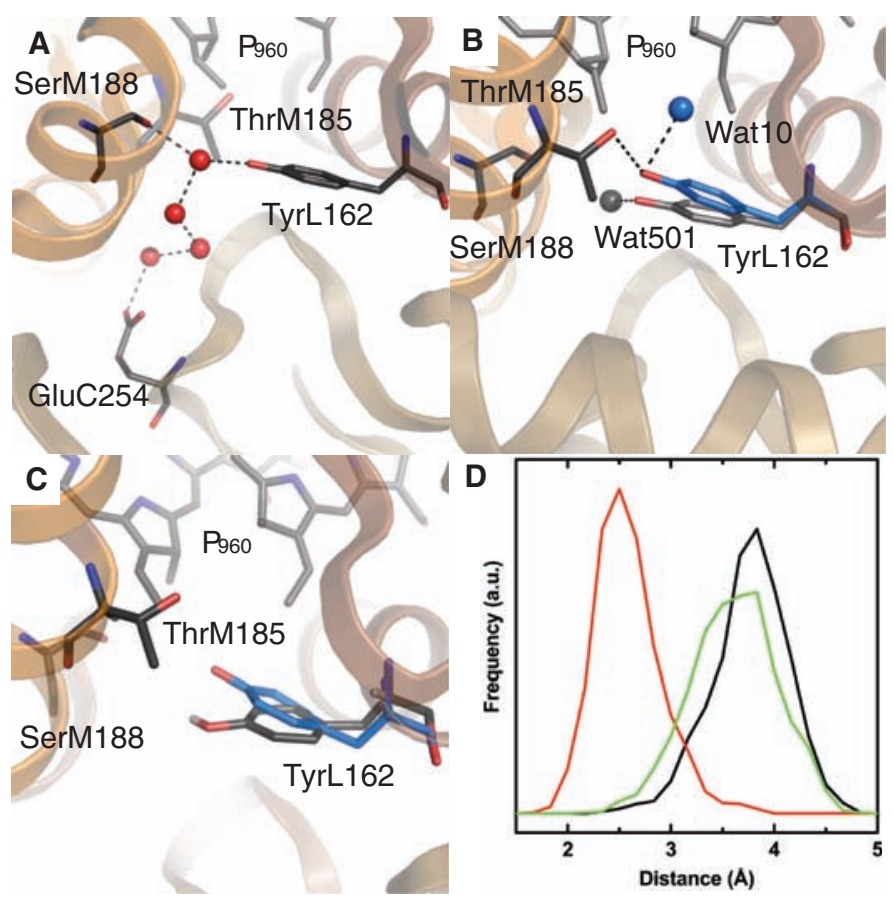
TyrL162

not when protonated (gray). (D) Histogram plot showing the frequency with which a given minimum distance from the TyrL162 side chain to the photooxidized special pair occurs when TyrL162 is protonated (green) or deprotonated (red) and for the nonilluminated state (black). Histograms were extracted from the final 30 ns (after 10-ns equilibration) of molecular dynamics trajectories.

Fig. 4. Structural overlay of photosystem II (green, PDB entry 1S5L) and $\mathrm{RC}_{\text {vir }}$ (brown) near the special pair. TyrL162 lies between the highpotential heme of the cytochrome c subunit (heme 3 ) and the special pair of $\mathrm{RC}_{\mathrm{vir}}$ (brown, $\mathrm{P}_{960}$ ), whereas $\mathrm{Tyr}_{\mathrm{z}}$ lies between the oxygen-evolving center (OEC) and the special pair of photosystem II (green). Arrows indicate the movement of electrons as the photooxidized special pair is reduced.

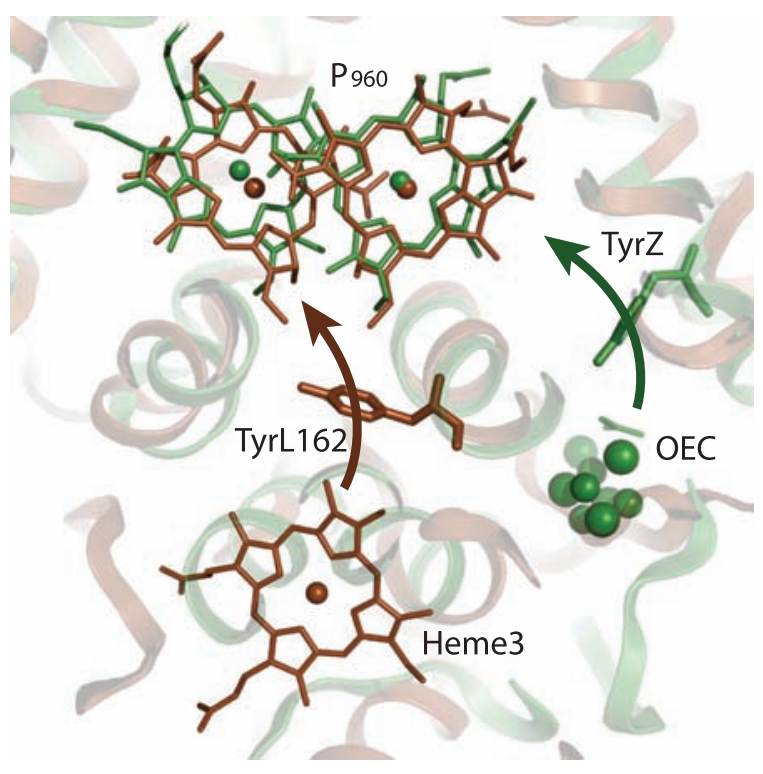

hydrogen bond changes of a tyrosine residue located near the special pair (13). Although this feature might also be taken to indicate TyrL162 deprotonation, its unique assignment is challenging because the prosthetic groups also contribute a strong difference signal in this region (14).

The free energy required for the transfer of the hydroxyl proton of TyrL162 to the carboxylate moiety of GluC254 (Fig. 3A) was also calculated from molecular dynamics trajectories of both the resting $\left(\mathrm{P}: \mathrm{Q}_{\mathrm{A}}\right)$ and the photoactivated $\left(\mathrm{P}^{+}: \mathrm{Q}_{\mathrm{A}}{ }^{-}\right)$ states with the tetraheme cytochrome $\mathrm{c}$ subunit both fully oxidized and fully reduced. From these trajectories, we observe that the energy requirements for proton transfer were lowered upon photoactivation by the same amount independent of whether the cytochrome subunit was oxidized or reduced $\left[46 \pm 3 \mathrm{~kJ} \mathrm{~mol}^{-1}\right.$ and $47 \pm 6$ $\mathrm{kJ} \mathrm{mol}^{-1}$, respectively (fig. S7)]. These values correspond to a relative change of $8 \pm 1 \mathrm{p} K_{\mathrm{a}}$ units (where $K_{\mathrm{a}}$ is the acid dissociation constant) such that, should the resting-state $\mathrm{p} K_{\mathrm{a}}$ values of TyrL162 and GluC254 be close to their reference values of 9.8 and 4.3 , respectively (15), spontaneous proton transfer would occur. Moreover, the water-mediated hydrogen bond network that connects these two groups (Fig. 3A and fig. S8) also provides an efficient channel for conducting a proton into the bulk $(\mathrm{pH}=8.1)$ via the solventexposed GluC254, such that TyrL152 could spontaneously deprotonate even if its resting $\mathrm{p} K_{\mathrm{a}}$ value was significantly elevated. In this manner, the excess positive charge on $\mathrm{P}^{+}$induces a proton transfer from TyrL162 in a direction opposite to that of the electron transfer, illustrating how coupled electron-proton transfer reactions arise in photosynthetic energy conversion. This principle could be translated into future synthetic systems for artificial photosynthesis $(16,17)$.

Spectroscopic studies of TyrL162 mutants of $\mathrm{RC}_{\mathrm{sph}}$ have established that this aromatic residue strongly influences the binding affinity of the soluble cytochrome $\mathrm{c}_{2}$ to photooxidized $\mathrm{RC}_{\mathrm{sph}}$ $(18,19)$, and this effect correlates with the kinetics of electron transfer to the special pair (19). For $\mathrm{RC}_{\mathrm{vir}}$, which has a permanently bound tetraheme cytochrome c subunit, the mutation of TyrL162 has considerably less effect on the rate of electron transfer from heme $\mathrm{c}_{559}$ to $\mathrm{P}^{+}(11)$, and the specific differences could be explained in terms of a super exchange mechanism with the residue at L162 mediating the electron transfer (20). This electron transfer reaction, however, is drastically inhibited below $215 \mathrm{~K}$ in the wildtype $\mathrm{RC}_{\mathrm{vir}}$, and both the extent of inhibition and its temperature dependence are strongly perturbed by mutation of TyrL162 (12). This remarkable temperature sensitivity has been interpreted in terms of a loss of mobility of TyrL162 at low temperature $(21,22)$ or resulting from the suppression of unspecified structural reorganizations associated with the oxidation of heme $\mathrm{c}_{559}$ (12). Our observation that TyrL162 changes conformation in response to photooxidation of $\mathrm{P}^{+}$adds credence to suggestions that the specific orienta- 
tion (21) and protonation state (22) of TyrL162 may be important for the mechanism of electron transfer, and the transient creation of a negatively charged tyrosyl group between heme $\mathrm{c}_{559}$ and $\mathrm{P}^{+}$ could stabilize the oxidized form of the cytochrome subunit (12).

From the temperature dependence of the $\mathrm{P}^{+}: \mathrm{Q}_{\mathrm{A}}^{-} \rightarrow \mathrm{P}: \mathrm{Q}_{\mathrm{A}}$ charge recombination reaction, it was proposed that structural changes occurring in response to electron transfer decrease the free energy gap between $\mathrm{P}^{+}$and $\mathrm{Q}_{\mathrm{A}}{ }^{-}$of $\mathrm{RC}_{\mathrm{sph}}$ by about $12 \mathrm{~kJ} \mathrm{~mol}^{-1}$ (23). Similar measurements of the $\mathrm{pH}$ dependence of the $\mathrm{RC}_{\mathrm{vir}}$ charge recombination reaction showed the $\mathrm{P}^{+}: \mathrm{Q}_{\mathrm{A}}^{-}$state to be stabilized by a chemical group with an approximate $\mathrm{p} K_{\mathrm{a}}$ of 9 (24). Prolonged exposure to bright light is also known to reversibly stabilize the $\mathrm{P}^{+}: \mathrm{Q}_{\mathrm{A}}{ }^{-}$state of $\mathrm{RC}_{\mathrm{sph}}$, slowing the charge recombination reaction by up to three orders of magnitude $(3,25,26)$. Light-induced deprotonation and conformational switching of TyrL162 could contribute to all three stabilization effects, because the creation of a phenolate anion in immediate proximity to the special pair effectively neutralizes the energetic penalty associated with the buried positive charge on $\mathrm{P}^{+}$.

Electron paramagnetic resonance spectroscopy studies of $\mathrm{RC}_{\mathrm{sph}}$ mutants that increase the midpoint potential of the special pair have shown that TyrL162 can form a (deprotonated) tyrosyl radical in response to charge separation (27), confirming that TyrL162 can be deprotonated by photooxidation of the special pair. Photooxidation of photosystem II also creates a tyrosyl radical $\left(\mathrm{Tyr}_{\mathrm{Z}}{ }^{\circ}\right)(28,29)$, which oxidizes the manganese cluster and leads to the synthesis of molecular oxygen from water. Crystal structures of photosystem II (30) reveal that $\mathrm{Tyr}_{Z}$ lies between the oxygen-evolving center and the special pair in a position functionally analogous to that occupied by TyrL162 of $\mathrm{RC}_{\mathrm{vir}}$ (Fig. 4). Our findings suggest that the deprotonation and coupled conformational switching of TyrL162 may have aided the spontaneous formation of tyrosine radicals in an ancient reaction center, thereby creating the chemical potential to extract electrons from clusters of manganese atoms (31) and ultimately oxidize water to oxygen.

References and Notes

1. J. Deisenhofer, O. Epp, K. Miki, R. Huber, H. Michel, Nature 318, 618 (1985).

2. M. H. Stowell et al., Science 276, 812 (1997).

3. G. Katona et al., Nat. Struct. Mol. Biol. 12, 630 (2005).

4. V. Srajer et al., Science 274, 1726 (1996).

5. F. Schotte et al., Science 300, 1944 (2003).

6. U. K. Genick et al., Science 275, 1471 (1997).

7. B. Perman et al., Science 279, 1946 (1998).

8. R. H. Baxter et al., Proc. Natl. Acad. Sci. U.S.A. 101 , 5982 (2004)

9. Materials and methods are available as supporting material on Science Online.

10. A. B. Wöhri et al., Biochemistry 48,9831 (2009).

11. B. Dohse et al., Biochemistry 34, 11335 (1995).

12. J. M. Ortega, B. Dohse, D. Oesterhelt, P. Mathis, Biophys. J. 74, 1135 (1998).
13. M. Leonhard, W. Mäntele, Biochemistry 32, 4532 (1993).

14. S. Buchanan, H. Michel, K. Gerwert, Biochemistry 31, 1314 (1992).

15. R. L. Thurlkill, G. R. Grimsley, ]. M. Scholtz, C. N. Pace, Protein Sci. 15, 1214 (2006).

16. N. S. Lewis, D. G. Nocera, Proc. Natl. Acad. Sci. U.S.A. 103, 15729 (2006).

17. L. Hammarström, S. Styring, Philos. Trans. R. Soc. London Ser. B 363, 1283 (2008)

18. J. Wachtveitl, ]. W. Farchaus, P. Mathis, D. Oesterhelt, Biochemistry 32, 10894 (1993).

19. X. M. Gong, M. L. Paddock, M. Y. Okamura, Biochemistry 42, 14492 (2003).

20. Y. Ohtsuka, K. Ohkawa, H. Nakatsuji, J. Comput. Chem. 22, 521 (2001)

21. B. Cartling, J. Chem. Phys. 95, 317 (1991).

22. B. Cartling, Chem. Phys. Lett. 196, 128 (1992).

23. B. H. McMahon, J. D. Müller, C. A. Wraight, G. U. Nienhaus, Biophys. J. 74, 2567 (1998).

24. P. Sebban, C. A. Wraight, Biochim. Biophys. Acta 974, 54 (1989).

25. F. van Mourik, M. Reus, A. R. Holzwarth, Biochim. Biophys. Acta 1504, 311 (2001).

26. U. Andréasson, L. E. Andréasson, Photosynth. Res. 75, 223 (2003).

27. A. J. Narváez, R. LoBrutto, J. P. Allen, J. C. Williams, Biochemistry 43, 14379 (2004).

28. B. A. Barry, G. T. Babcock, Proc. Natl. Acad. Sci. U.S.A. 84, 7099 (1987).
29. R. J. Debus, B. A. Barry, I. Sithole, G. T. Babcock, L. McIntosh, Biochemistry 27, 9071 (1988)

30. K. N. Ferreira, T. M. Iverson, K. Maghlaoui, J. Barber, S. Iwata, Science 303, 1831 (2004); published online 5 February 2004 (10.1126/science.1093087).

31. J. F. Allen, W. Martin, Nature 445, 610 (2007).

32. Refined crystallographic coordinates and structure factor amplitudes are deposited within the Protein Data Bank (PDB) with codes $2 \times 5 \mathrm{u}$ (dark state) and $2 \times 5 \mathrm{v}$ (photoactivated state). We thank L. Hammarström for valuable discussions and acknowledge financial support from the Swedish Science Research Council (Vetenskapsrådet), European Commission [Research Training Network (RTN) "Fast light-actuated structural changes" (FLASH), and Integrated Project, the European Membrane Protein Consortium (EMEP)], European Molecular Biology Organization (EMBO), Human Frontier Science Program (HFSP), and the University of Gothenburg Quantitative Biology Platform.

\section{Supporting Online Material}

www.sciencemag.org/cgi/content/full/328/5978/630/DC1

Materials and Methods

Figs. S1 to S10

Tables $\mathrm{S} 1$ to $\mathrm{S3}$

Scheme S1

References

21 December 2009; accepted 17 March 2010

10.1126/science.1186159

\section{The Genome of the Western Clawed Frog Xenopus tropicalis}

Uffe Hellsten, ${ }^{1 *}$ Richard M. Harland, ${ }^{2}$ Michael J. Gilchrist, ${ }^{3}$ David Hendrix,${ }^{2}$ Jerzy Jurka, ${ }^{4}$ Vladimir Kapitonov, ${ }^{4}$ Ivan Ovcharenko, ${ }^{5}$ Nicholas H. Putnam, ${ }^{6}$ Shengqiang Shu, ${ }_{1}^{1}$ Leila Taher, ${ }^{5}$ Ira L. Blitz, ${ }^{7}$ Bruce Blumberg, ${ }^{7}$ Darwin S. Dichmann, ${ }^{2}$ Inna Dubchak, ${ }^{1}$ Enrique Amaya, ${ }^{8}$ John C. Detter, ${ }^{9}$ Russell Fletcher, ${ }^{2}$ Daniela S. Gerhard, ${ }^{10}$ David Goodstein, ${ }^{1}$ Tina Graves, ${ }^{11}$ Igor V. Grigoriev, ${ }_{1}^{1}$ ]ane Grimwood, ${ }^{1,12}{ }^{\text {Takeshi Kawashima, }}{ }^{2,13}$ Erika Lindquist, ${ }^{1}$ Susan M. Lucas, ${ }^{1}$ Paul E. Mead ${ }^{14}$ Therese Mitros, ${ }^{2}$ Hajime Ogino ${ }^{15}$ Yuko Ohta, ${ }^{16}$ Alexander V. Poliakov, ${ }^{1}$ Nicolas Pollet, ${ }^{17}$ Jacques Robert, ${ }^{18}$ Asaf Salamov ${ }_{,}{ }^{1}$ Amy K. Sater, ${ }^{19}$ Jeremy Schmutz, ${ }^{1,12}$ Astrid Terry, ${ }^{1}$ Peter D. Vize, ${ }^{20}$ Wesley C. Warren, ${ }^{11}$ Dan Wells, ${ }^{19}$ Andrea Wills, ${ }^{2}$ Richard K. Wilson, ${ }^{11}$ Lyle B. Zimmerman, ${ }^{21}$ Aaron M. Zorn, ${ }^{22}$ Robert Grainger, ${ }^{23}$ Timothy Grammer, $^{2}$ Mustafa K. Khokha, ${ }^{24}$ Paul M. Richardson, ${ }^{1}$ Daniel S. Rokhsar ${ }^{1,2}$

The western clawed frog Xenopus tropicalis is an important model for vertebrate development that combines experimental advantages of the African clawed frog Xenopus laevis with more tractable genetics. Here we present a draft genome sequence assembly of $X$. tropicalis. This genome encodes more than 20,000 protein-coding genes, including orthologs of at least 1700 human disease genes. Over 1 million expressed sequence tags validated the annotation. More than one-third of the genome consists of transposable elements, with unusually prevalent DNA transposons. Like that of other tetrapods, the genome of $X$. tropicalis contains gene deserts enriched for conserved noncoding elements. The genome exhibits substantial shared synteny with human and chicken over major parts of large chromosomes, broken by lineage-specific chromosome fusions and fissions, mainly in the mammalian lineage.

A frican clawed frogs (the genus Xenopus, meaning "strange foot") comprise more than 20 species of frogs native to SubSaharan Africa. The species Xenopus laevis was first introduced to the United States in the 1940s where a low-cost pregnancy test took advantage of the responsiveness of frogs to human chorionic gonadotropin (1). Since the frogs were easy to raise and had other desirable properties such as large eggs, external development, easily manipulated embryos, and transparent tadpoles, $X$. laevis gradually developed into one of the most productive model systems for vertebrate experimental embryology (2).

However, $X$. laevis has a large paleotetraploid genome with an estimated size of 3.1 billion bases (Gbp) on 18 chromosomes and a generation time of 1 to 2 years. In contrast, the much smaller diploid western clawed frog, $X$. tropicalis, has a small genome, about $1.7 \mathrm{Gbp}$ on 10 chromosomes (3), matures in only 4 months, and requires less space than its larger cousin. It is thus 